\title{
First-line chemotherapy with docetaxel plus capecitabine followed by capecitabine or hormone maintenance therapy for the treatment of metastatic breast cancer patients
}

\author{
XU LIANG, YING YAN, LINA WANG, GUOHONG SONG, LIJUN DI, \\ HANFANG JIANG, CHAOYING WANG and HUIPING LI \\ Key Laboratory of Carcinogenesis and Translational Research (Ministry of Education), Department of Breast Oncology, \\ Peking University Cancer Hospital and Institute, Beijing 100142, P.R. China
}

Received May 23, 2014; Accepted December 1, 2014

DOI: $10.3892 / \mathrm{ol} .2014 .2787$

\begin{abstract}
The primary aim of the present study was to evaluate whether maintenance therapy with capecitabine or hormone replacement therapy (HRT) results in improved progression-free survival (PFS) in metastatic breast cancer (MBC) patients who had previously achieved disease control with first-line docetaxel plus capecitabine (TX) chemotherapy. Seventy-nine metastatic breast cancer patients treated between January 2008 and June 2013 with TX chemotherapy were retrospectively analyzed. Following successful initial disease control by the combination chemotherapy, 39 patients received single-agent capecitabine maintenance therapy and 40 patients received HRT as maintenance therapy. The PFS time, objective response rate, clinical benefit rate and safety of the two groups were compared. The median PFS of the total cohort $(n=79)$ was 11.0 months. Furthermore, the median PFS time of the capecitabine $(n=39)$ and HRT groups $(n=40)$ were 10.9 and 11.1 months, respectively $(\mathrm{P}=0.283)$. Compared with the PFS time of maintenance treatment only, single-agent capecitabine treatment following TX chemotherapy prolonged the PFS time by 6.8 months and HRT following TX chemotherapy prolonged PFS time by 5.8 months $(\mathrm{P}=0.551)$. Of the total cohort, 49 patients did not receive palliative endocrine therapy prior to chemotherapy, including 22 patients in the capecitabine maintenance group and 27 patients in the HRT maintenance group. The PFS time from the commencement of maintenance treatment was significantly different between the two groups, 6.1 months in the capecitabine group compared with 11.5 months in the HRT group $(\mathrm{P}=0.045)$. For the 30 patients who underwent palliative endocrine therapy prior
\end{abstract}

Correspondence to: Dr Huiping Li, Department of Breast Oncology, Beijing Cancer Hospital, Peking University Cancer Hospital and Institute, 52 Fucheng Road, Beijing 100142, P.R. China E-mail: huipingli2013@yahoo.com

Key words: capecitabine, maintenance therapy, docetaxel, metastatic breast cancer to TX chemotherapy, the PFS times of the capecitabine and HRT maintenance treatment groups were 7.5 and 4.1 months, respectively $(\mathrm{P}=0.043)$. However, the occurrence of adverse events, such as hematological and gastrointestinal toxicity, as well as hand-foot syndrome, were not significantly different between the two groups. The current study indicated that single-agent capecitabine maintenance therapy may be a potential treatment strategy for MBC patients who responded to capecitabine-based chemotherapy. In particular, capecitabine may provide a more effective maintenance treatment duration compared with HRT for patients who had previously undergone first-line palliative HRT for MBC.

\section{Introduction}

Breast cancer represents the most common type of malignancy in females, worldwide. Despite earlier diagnosis and improvement in adjuvant therapies, a number of patients present with metastatic recurrence, which has a two to three year median overall survival time $(1,2)$. Hormonal therapy, chemotherapy and more recently biological treatment are systemic therapies designed to reduce the size of tumors, improve patient survival and preserve quality of life. However, in a metastatic setting, the majority of patients will relapse regardless of the initial efficacy of the treatment strategy undertaken. The most important therapeutic goals in metastatic breast cancer (MBC) are palliative and aim to improve progression free survival (PFS). However, this management of MBC is a clinical challenge for healthcare workers, as the optimal type and duration of chemotherapy, and the benefits of maintenance chemotherapy versus maintenance hormonal treatment required, have yet to be determined. Thus, the present retrospective study aimed to investigate the impact of HRT and capecitabin, two types of maintenance therapy, on MBC patient PFS.

Following a response to rescue chemotherapy, maintenance treatment with HRT or targeted agents may be considered for the treatment of MBC; however, maintenance HRT is limited to $\mathrm{MBC}$ patients with hormone receptor-positive disease $(3,4)$. A number of targeted agents are widely accepted as a type of maintenance therapy for MBC, for example trastuzumab is administered for human epidermal growth factor receptor 2 
(Her-2)-positive MBC (5,6). However, targeted agents are relatively high in cost and, thus, are not routinely selected as maintenance treatment in developing countries. In addition to efficacy, the convenience and tolerability of the maintenance treatment must be considered; for example, intravenous chemotherapy requires frequent hospital visits for the patient, which are associated decreased quality of life for patients and increased healthcare worker costs. Therefore, the majority of patients prefer oral as opossed to intravenous chemotherapy $(7,8)$, for example oral capecitabine.

Capecitabine is approved by the US Food and Drug Administration for the treatment of patients with locally advanced breast cancer or MBC. It has a favorable safety profile with adverse events effectively managed by dose modification (9) and it can conveniently be administered by oral dosing (10). Furthermore, capecitabine typically lacks cumulative toxicity with prolonged use and, thus, is suitable for long-term administration. A number of clinical trials of capecitabine for the treatment of MBC indicate that capecitabine is effective when combined with a variety of agents, including taxanes, vinorelbine, gemcitabine, trastuzumab or bevacizumab (11-16). However, it is unclear how the therapeutic effects of capecitabine-based first-line combination chemotherapy may be maintained. Thus, the current study presents the results of an analysis of $\mathrm{MBC}$ patients receiving capecitabine or hormone replacement therapy (HRT) as maintenance treatment following initial response to capecitabine-based combination therapy.

\section{Patients and methods}

Patient selection. From January 2008 to June 2013, 226 MBC patients received TX combination therapy at the Department of Breast Oncology of Beijing Cancer Hospital (Beijing, China). Of these, 79 patients were eligible to receive maintenance treatment according to the following inclusion criteria: Female patients aged $\geq 18$ years with histologically confirmed primary breast cancer; patients must have a minimum of one measurable lesion, according to Response Evaluation Criteria in Solid Tumors guidelines (RECIST) 1.0 (17), and an Eastern Cooperative Oncology Group score of $\leq 2$ (18); patients must not have undergone prior chemotherapy for advanced disease; and patients must have completed four to eight cycles and achieved disease control [complete relief (CR), partial relief (PR) or stable disease (SD)]. Furthermore, patients were allowed to receive one-line endocrine treatment for advanced disease prior to docetaxel plus capexitabine (TX) chemotherapy. This study was approved by the ethics committee of Beijing Cancer Hospital (Beijing, China) and written informed consent was obtained from all patients.

Treatment strategy. Capexitabine was administered as the combination and maintenance therapy at a dose of $1,000 \mathrm{mg} / \mathrm{m}^{2}$ twice daily on days 1-14 followed by a 7-day rest period. In the combination regimen, docetaxel was coadministered, as a 1 -h $75 \mathrm{mg} / \mathrm{m}^{2}$ intravenous infusion on day 1 of every 3 -week cycle. Following a response to chemotherapy, 39 patients continued to receive single-agent capecitabine with the abovementioned dose, whilst 40 patients received hormonal therapy with tamoxifen $(n=3)$, toremifene $(n=7)$, exemestane $(n=15)$, letrozle $(n=6)$ or anastrozole $(n=9)$. To relieve the symptoms of hand-foot syndrome during maintenance therapy, all patients were coadministered with $100 \mathrm{mg}$ vitamin B6 three times daily.

Efficacy and safety assessments. The PFS time of the 79 patients was determined as the interval from the day of combined TX chemotherapy commencement to cancer progression, cancer-related mortality, mortality from an unknown cause during therapy, or the final day of follow-up for patients who had not progressed at the date of analysis. By contrast, for maintenance treatment PFS, the start time was defined as the day of capecitabine or hormonal agent maintenance therapy commencement. Additionally, the clinical efficacy and major adverse events were investigated, with response assessed using RECIST and adverse events graded according to the National Cancer Institute Common Toxicity Criteria version 3.0 (19).

Statistical analysis. The duration of response was defined as the period between CR or PR onset and evidence of disease progression, and the duration of response and PFS were estimated using the Kaplan-Meier method. Additionally, the baseline characteristics of the patients and the incidence of adverse events between capecitabine and HRT maintenance therapy were compared using Pearson's $\chi^{2}$ test. All statistical analyses were performed using SPSS software (version 15.0; SPSS Inc., Chicago, IL, USA). P $<0.05$ was considered to indicate a statistically significant difference.

\section{Results}

Patient characteristics. The 79 patients investigated in the present study were divided into two groups, with 39 patients receiving capecitabine maintenance therapy and 40 patients receiving hormone maintenance therapy. The baseline patient characteristics of the 79 patients are summarized in Table I. The median patient age was 55 years (range, 34-75 years), the majority of patients exhibited hormone receptor-positive tumors $(79.8 \%$; HR-positive status indicates estrogen receptor-positive and/or progesterone receptor-positive), and Her-2-negative disease (83.5\%). The most common sites of metastasis were the bone and lung (51.9\%). The majority of patients had received prior anthracycline-based chemotherapy $(78.4 \%)$, with more than half $(50.6 \%)$ receiving prior taxane-based chemotherapy. Additionally, palliative hormonal therapy due to metastasis had been administered prior to DX chemotherapy in 30 patients, including 17 patients $(43.5 \%)$ in the capecitabine maintenance group and 13 patients (32.5\%) in the HRT group. Of the 40 patients who received endocrine agent maintenance, 28 patients received aromatase inhibitors (AIs), five patients received toremifene, six received goserelin plus AIs and one patient received tamoxifene.

Efficacy of combined DX chemotherapy plus maintenance treatment. Combined agents chemotherapy plus maintenance therapy was received by all 79 patients and resulted in a median PFS of 11.0 months [95\% confidence interval (CI), 10.1-11.9 months; Fig. 1]. Dependent on the nonprogressive response, eight patients $(10.1 \%)$ received eight cycles 
Table I. Baseline characteristics of all patients $(n=79)$.

\begin{tabular}{|c|c|c|c|c|c|}
\hline \multirow[b]{2}{*}{ Characteristics } & \multicolumn{2}{|c|}{ Capecitabine maintenance } & \multicolumn{2}{|c|}{ HRT maintenance } & \multirow[b]{2}{*}{ P-value } \\
\hline & $\mathrm{n}$ & $\%$ & $\mathrm{n}$ & $\%$ & \\
\hline Menopause status & & & & & 0.406 \\
\hline Pre & 12 & 30.8 & 9 & 22.5 & \\
\hline Post & 27 & 69.2 & 31 & 77.5 & \\
\hline ECOG PS & & & & & 0.372 \\
\hline 0 & 23 & 59.0 & 30 & 75.0 & \\
\hline 1 & 14 & 35.9 & 9 & 22.5 & \\
\hline 2 & 2 & 5.1 & 1 & 2.5 & \\
\hline HR status & & & & & 0.082 \\
\hline Positive & 28 & 71.8 & 35 & 87.5 & \\
\hline Negetive & 11 & 28.2 & 5 & 12.5 & \\
\hline Lymph nodes, $\mathrm{n}^{\mathrm{a}}$ & & & & & 0.516 \\
\hline $0-3$ & 28 & 71.8 & 26 & 65.0 & \\
\hline$\geq 4$ & 11 & 28.2 & 14 & 35.0 & \\
\hline Her-2 status & & & & & 0.876 \\
\hline Positive $^{\mathrm{b}}$ & 6 & 15.4 & 5 & 12.5 & \\
\hline Negative $^{c}$ & 32 & 82.1 & 34 & 85.0 & \\
\hline Unknown & 1 & 2,4 & 1 & 2.5 & \\
\hline \multicolumn{6}{|l|}{ Metastatic site } \\
\hline Liver & 14 & 35.9 & 12 & 30.0 & 0.577 \\
\hline Lung & 23 & 59.0 & 18 & 45.0 & 0.214 \\
\hline Bone & 20 & 51.3 & 21 & 52.5 & 0.914 \\
\hline Brain & 3 & 7.7 & 4 & 10 & 1.000 \\
\hline Soft tissue & 22 & 56.4 & 30 & 75.0 & 0.082 \\
\hline Visceral metastasis & & & & & 0.210 \\
\hline Yes & 32 & 82.1 & 28 & 70.0 & \\
\hline No & 7 & 17.9 & 12 & 30.0 & \\
\hline Metastatic sites, $\mathrm{n}$ & & & & & 0.943 \\
\hline 1 & 7 & 17.9 & 8 & 20.0 & \\
\hline 2 & 18 & 46.2 & 17 & 42.5 & \\
\hline$\geq 3$ & 14 & 35.9 & 15 & 37.5 & \\
\hline Disease-free interval, years & & & & & 0.539 \\
\hline$<2$ & 13 & 33.3 & 16 & 40.0 & \\
\hline$\geq 2$ & 26 & 66.7 & 24 & 60.0 & \\
\hline Prior adjuvant chemotherapy & & & & & 0.523 \\
\hline Taxane & 20 & 51.3 & 20 & 50.0 & \\
\hline Anthracycline & 35 & 89.7 & 27 & 67.5 & \\
\hline Prior adjuvant endocrine therapy & 25 & 64.1 & 27 & 67.5 & 0.764 \\
\hline Prior palliative endocrine therapy & 17 & 43.5 & 13 & 32.5 & 0.310 \\
\hline
\end{tabular}

of combined chemotherapy, 14 patients (17.7\%) received four cycles and 57 patients $(72.2 \%)$ recevied six cycles. The baseline response to the combination chemotherapy was a CR in two patients (2.5\%), a PR in 32 patients $(40.5 \%)$ and $\mathrm{SD}$ in 45 patients $(57.0 \%)$. For the 39 patients following the single-agent capecitabine maintenance treatment, the baseline was as follows: Two patients (5.1\%) achieved a CR, 20 patients $(51.3 \%)$ exhibited SD and PR occured in 17 patients $(43.6 \%)$, whilst in the $40 \mathrm{HRT}$ patients, PR occured in 15 patients $(37.5 \%)$ and SD in 25 patients $(62.5 \%)$. 
Table II. Treatment-associated toxicities.

\begin{tabular}{|c|c|c|c|}
\hline Adverse event & $\begin{array}{c}\text { Capecitabine maintenance, } \\
\mathrm{n}(\%)\end{array}$ & $\begin{array}{l}\text { HRT maintenance, } \\
\mathrm{n}(\%)\end{array}$ & P-value \\
\hline Neutropenia, grade & & & 0.492 \\
\hline 0 & 17 (43.6) & $23(57.5)$ & \\
\hline 1 & $4(10.3)$ & $3(7.5)$ & \\
\hline 2 & $10(25.6)$ & $10(25.0)$ & \\
\hline 3 & $8(20.5)$ & $4(10.0)$ & \\
\hline 4 & $0(0.0)$ & $0(0.0)$ & \\
\hline Vomiting/diarrhea, grade & & & 0.433 \\
\hline 0 & 27 (69.2) & $29(72.5)$ & \\
\hline 1 & $6(15.4)$ & 7 (17.5) & \\
\hline 2 & $3(7.7)$ & $4(10.0)$ & \\
\hline 3 & $3(7.7)$ & $0(0.0)$ & \\
\hline 4 & $0(0.0)$ & $0(0.0)$ & \\
\hline Hand-foot syndrome, grade & & & $0.052^{\mathrm{a}}$ \\
\hline 0 & $20(51.3)$ & $29(72.5)$ & \\
\hline 1 & 7 (17.9) & $3(7.5)$ & \\
\hline 2 & $4(10.3)$ & $2(5.0)$ & \\
\hline 3 & $8(20.5)$ & $6(15.0)$ & $0.521^{b}$ \\
\hline
\end{tabular}

HRT, hormone replacement therapy. ${ }^{\mathrm{a}}$ Mean incidence of hand-foot syndrome (48.7 vs. $27.5 \%$ ). ${ }^{\mathrm{b}}$ Mean incidence of grade III toxicity hand-foot syndrome.

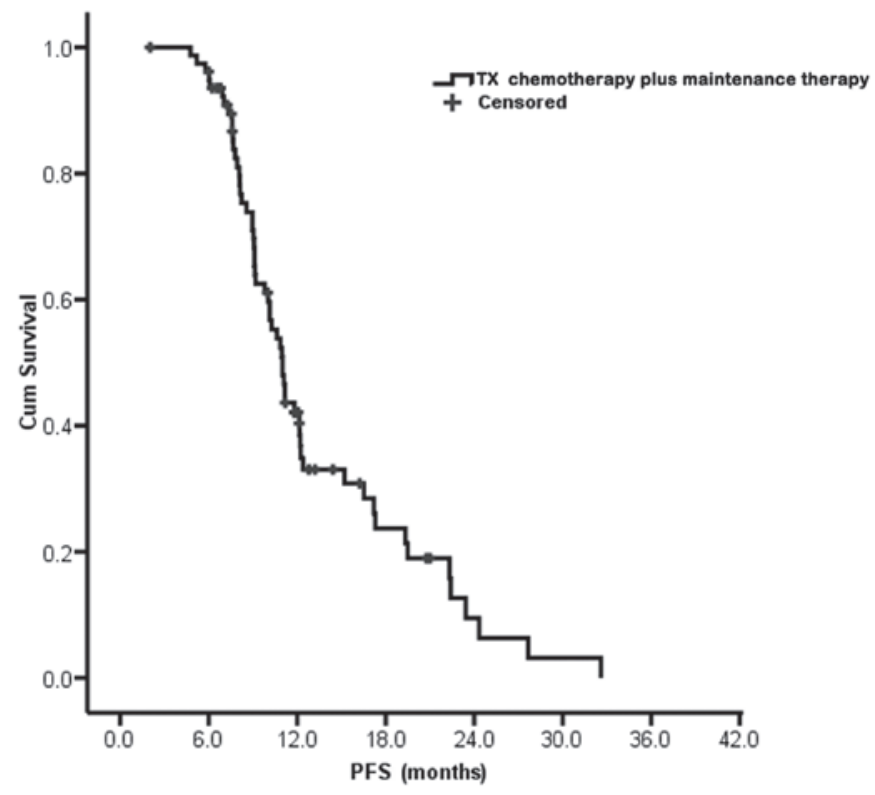

Figure 1. Median PFS of 79 patients who underwent combined chemotherapy followed by maintenance treatment. PFS, progression-free survival; $\mathrm{XD}$, docetaxel plus capecitabine.

The rate of CR and PR were not significantly different between the two groups (48.7 vs. $37.5 \%$, respectively; $\mathrm{P}=0.314$ ).

Efficacy of capecitabine maintenance therapy and HRT. The median PFS time of patients in the TX chemotherapy plus capecitabine maintenance therapy group was 10.9 months (95\% CI, 9.9-12.0 months) and for the TX chemotherapy plus
HRT group was 11.1 months (95\% CI, 8.8-13.4 months; $\mathrm{P}=0.28$; Fig. 2). Compared with the PFS time of maintenance treatment only, TX chemotherapy plus single-agent capecitabine treatment prolonged survival by 6.8 months (95\% CI, 5.7-7.9 months), which was not significantly different to the PFS time of TX chemotherapy plus HRT (5.8 months; 95\% CI, 4.0-7.6 months; $\mathrm{P}=0.55$; Fig. 3). The 6-month PFS rate of the two types of maintenance treatment were similar $(95 \% \mathrm{CI}, 51.3$ for capecitabine vs. $42.5 \%$ for HRT; $\mathrm{P}=0.434$ ).

Efficacy of maintenance therapy with or without palliative endocrine therapy prior to chemotherapy. In 49 patients, the first-line treatment strategy was not palliative hormonal therapy; this included 22 patients in the capecitabine maintenance group and 27 patients in the HRT maintenance group. For these 49 patients, the median PFS time from maintenance treatment was 6.1 months in the capecitabine group and 11.5 months in the HRT group ( $\mathrm{P}=0.045$; Fig. 4). Prior to the administration of TX chemotherapy, 30 patients had received palliative hormonal therapy as first-line therapy for metastatic breast cancer, including 17 patients in the capecitabine maintenance group and 13 in the HRT group. The median PFS time of these 30 patients from maintenance treatment was 7.5 months in the capecitabine group and 4.1 months in the HRT group $(\mathrm{P}=0.043$; Fig. 5). Furthermore, the 6-month PFS rate was $58.8 \%$ in the capecitabine maintenance group and $30.7 \%$ in the HRT group ( $\mathrm{P}=0.159$; Fig. 5). No significant difference was identified between the two maintenance groups; however, this may have been due to an insufficient number of cases being investigated, as it was observed that the 6-month PFS rate of the capecitabine maintenance group was almost twice that of the HRT group. 


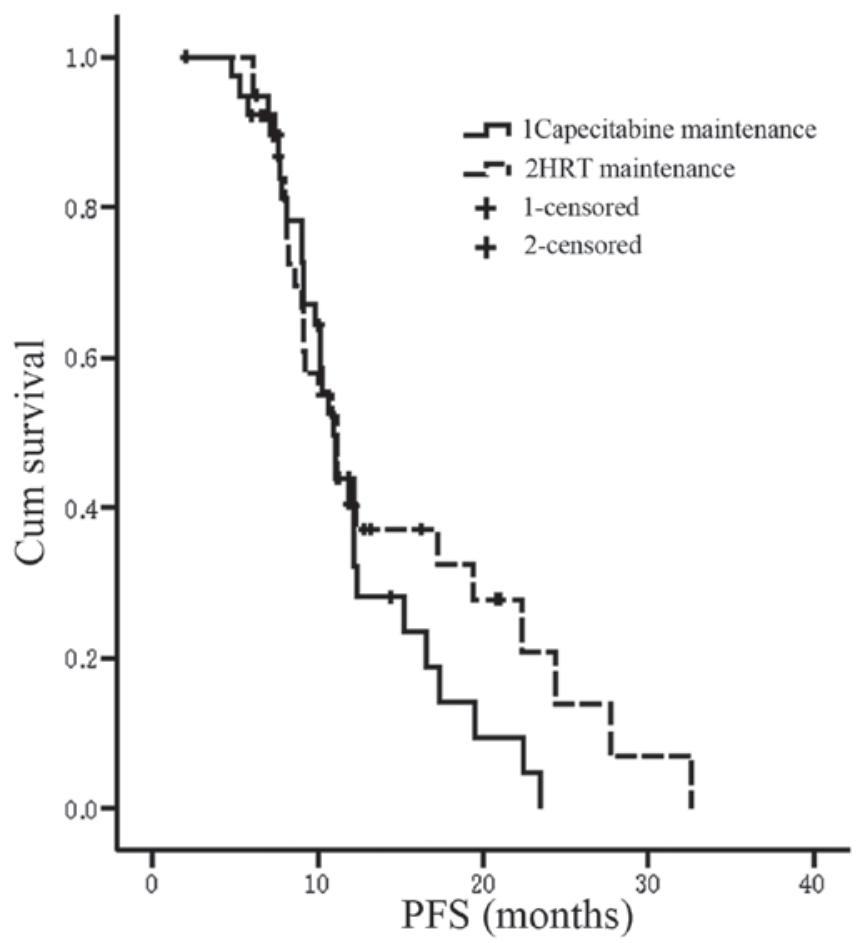

Figure 2. PFS of docetaxel plus capecitabine chemotherapy followed by two types of maintenance therapy. HRT, hormone replacement therapy; PFS, progression-free survival.

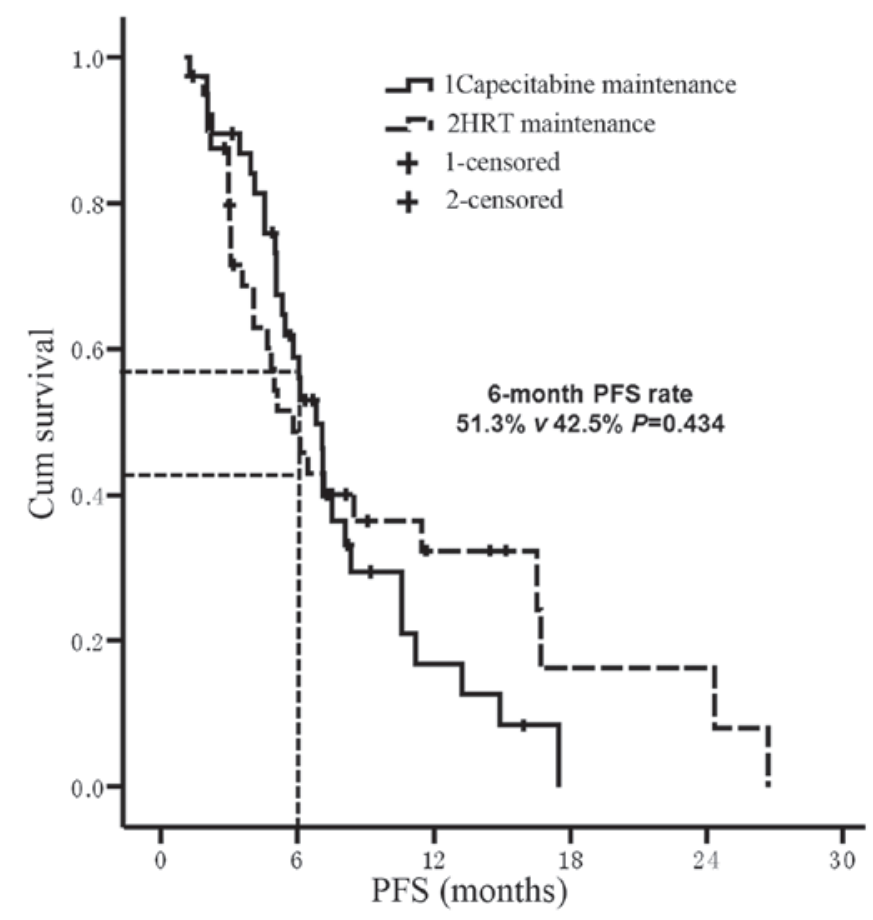

Figure 3. PFS of maintenance therapy in two groups. HRT, hormone replacement therapy; PFS, progression-free survival.

Toxicity analysis. Table II indicates the treatment-associated toxicities [according to National Cancer Institute Common Terminology Criteria for Adverse Events (19)] of 79 patients observed in the present study. Hematologic and gastrointestinal toxicities, as well as hand-foot syndrome did not occur at significantly different rates in the two groups. For

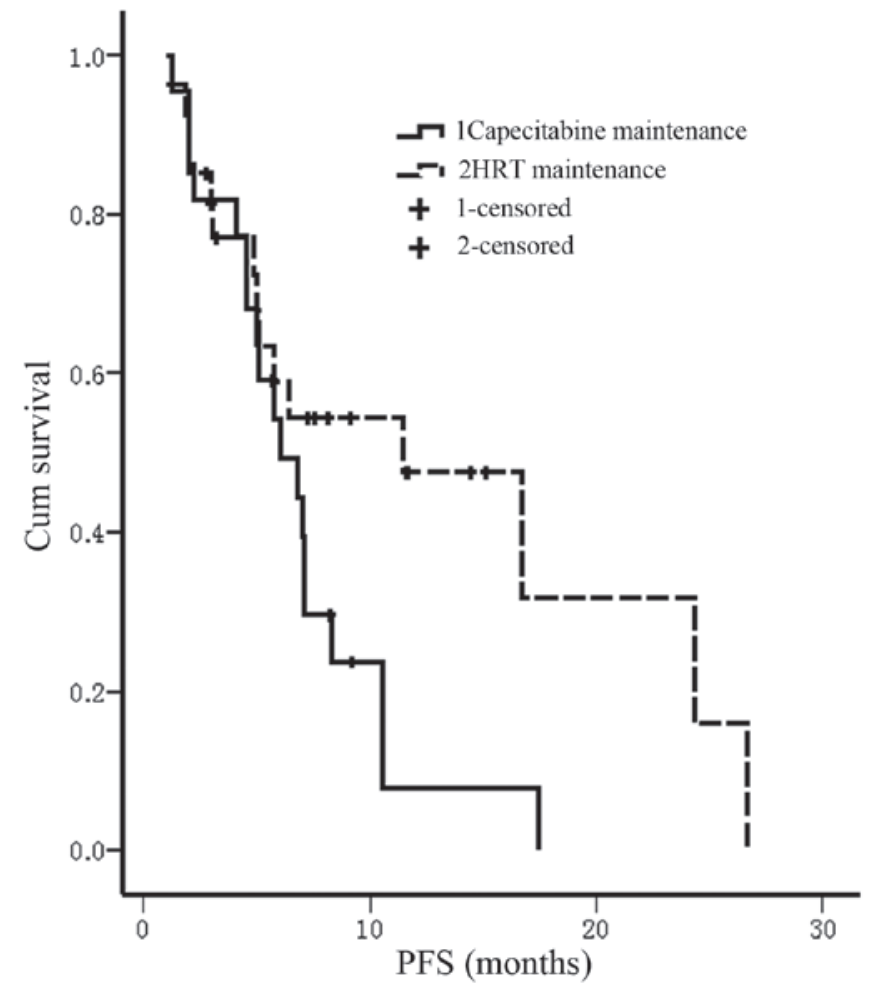

Figure 4. PFS of maintenance therapy for patients who did not undergo palliative hormonal therapy as the first-line therapy. HRT, hormone replacement therapy; PFS, progression-free survival.

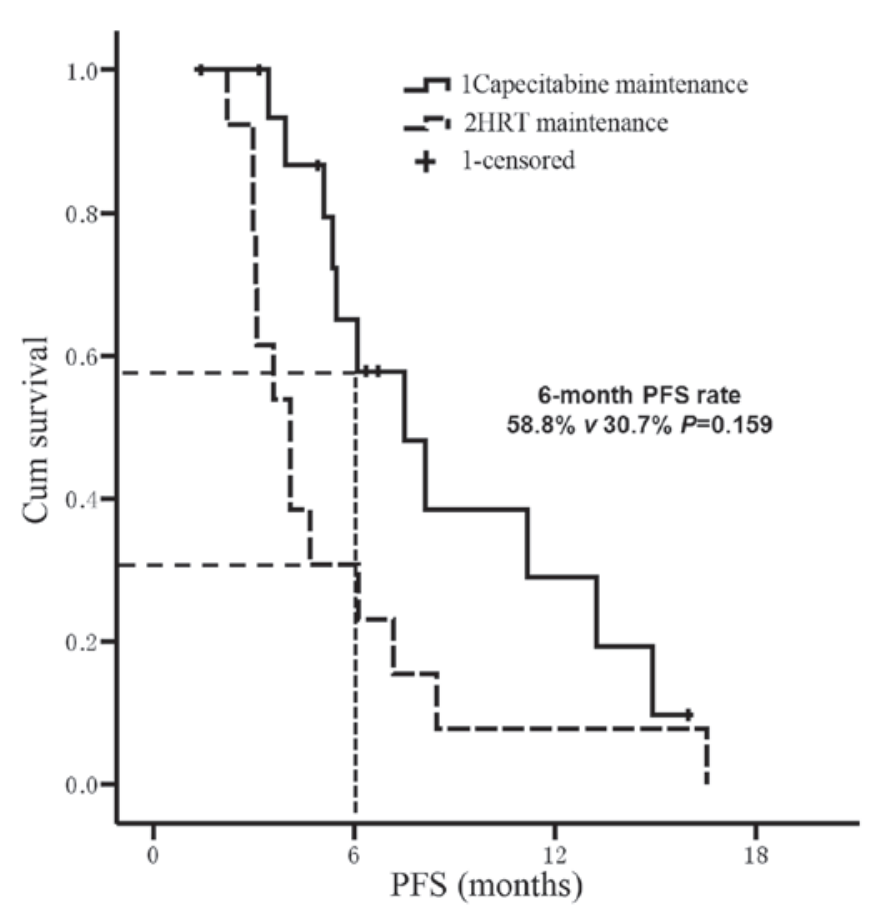

Figure 5. PFS of maintenance therapy for patients with palliative hormonal therapy as the first-line therapy. HRT, hormone replacement therapy; PFS, progression-free survival.

example, the rate of grade III neutropenia was marginally higher in the capecitabine maintenance group compared with the HRT group ( 20.5 vs. $10.0 \%$, respectively; $\mathrm{P}=0.225$ ), and the mean incidence of hand-foot syndrome was markedly 
greater in the capecitabine group compared with the HRT group (48.7 vs. $27.5 \%$, respectively; $\mathrm{P}=0.052$ ).

\section{Discussion}

The long-term survival of female MBC patients remains poor, despite decades of research into systemic therapy (20). Systemic therapy uses chemotherapy or hormonal therapy, depending on factors, such as hormone receptor status, performance status, disease bulk, number of disease sites and patient age. For HR-positive patients, initial chemotherapy may be selected as the treatment modality due to the aggressive nature of the disease; in particular, combination chemotherapy has demonstrated a number of potential benefits, including an increased therapeutic response, a shorter time to progression and the possibility of improved overall survival. Thus, chemotherapy is often selected as the the priority treatment strategy in patients exhibiting visceral metastasis (21). However, upon the termination of chemotherapy for metastatic disease, disease progression occurs quickly. For example, studies conducted by Park et al (22) and Alba et al (4) demonstrated that the median PFS time following chemotherapy termination was 3.8 and 5.1 months, respectively. Therefore, it is important that maintenance therapy for MBC patients is conducted. If a patient exhibits a hormone receptor-positive tumor, the majority of healthcare workers would initiate treatment with maintenance hormonal therapy following the completion of chemotherapy, despite the lack of prospective randomized trials regarding its efficacy (23). However, for patients with HR-negative tumors, endocrine-resistant disease of the luminal subtype or rapidly proliferative and/or symptomatic disease, there is no preferred method for maintaining stable disease. Recently, the Korean Cancer Study Group conducted a phase III clinical trial of HER2-negative MBC patients who had achieved disease control following six cycles of first-line paclitaxel/gemcitabine chemotherapy (22). The study determined that subsequent gemcitabine/paclitaxel maintenance chemotherapy was associated with a statistically significant increase in the median and 6-month PFS rates, as well as an increase in the overall survival period (22). Furthermore, single-agent chemotherapy was considered to be an effective maintenance treatment and was the preferred choice compared with combination agents.

The present study considered DX chemotherapy to be the preferred treatment stratetgy for MBC patients due to its positive response and tolerable side effects. In 2002, O'Shaughnessy et al (14) conducted a phase III study comparing the effects of docetaxel administraton alone with docetaxel in combination with capecitabine (TX chemotherapy). The addition of capecitabine to docetaxe treatment resulted in an extended time to disease progression, improved overall survival and more manageable side effects. Similarly, a PFS time of 11 months for TX chemotherapy was determined in the present study. Additionally, the total and maintenance PFS times were similar between the capecitabine and HRT maintenence groups (10.9 and 6.8 months vs. 11.1 and 5.8 months). Approximately half of the patients maintained their response to combination chemotherapy for $>6$ months and achieved a clinical benefit in regardless of whether they were in the capecitabine or HRT maintenence group; however, six patients received HRT for maintenance treatment $>12$ months and two patients for $>20$ months, while four patients received capecitabine $>1$ year. The improved response in the HRT group may be because HRT is better tolerated compared with capecitabine. For the 49 patients who did not undergo palliative endocrine therapy, the use of HRT for maintenance therapy demonstrated a longer PFS time (11.5 months vs. 6.1 months), consistent with previous reports $(6,24)$. Additionally, of the 30 patients who received HRT as first-line metastasis treatment prior to TX chemotherapy administration, the capecitabine maintenance group exhibited a higher PFS compared with the HRT maintenence group. This significant reduction in PFS $(\mathrm{P}=0.043)$ may be associated with endocrine resistance caused by repeated HRT (25-27). In the present study, 70\% patients were postmenopausal; and according to the results of several clinical trials, postmenopausal advanced breast cancer patients are initially recommended to undergo endocrine therapy predominantly consisting of a nonsteroidal (letrozole or anastrozole) or steroidal (exemestane) aromatase inhibitor (28-30). However, even if this type of hormonal therapy is initially effective, it considered to be ineffective following relapse caused by acquired resistance (26). According to the results of the present study, capecitabine may be an optional maintenance treatment for patients who are resistant to endocrine therapy.

Numerous trials have been conducted that indicate that the use of continuous chemotherapy for the treatment of breast cancer prolongs the duration of remission; however, its effect on quality of life and survival are less consistent $(3,31,32)$. Recently, a meta-analysis was conducted, which analyzed the data from 11 randomized trials. A longer duration period of first-line chemotherapy was associated with a markedly improved PFS period (5); however, it is essential that the appropriate agent is selected for maintenance treatment by considering its impact on quality of life and the extent of toxicity, against the improvement in disease-associated symptoms and the benefits of tumor regression. Using these considerations, capecitabine was selected as an appropriate candidate agent for patients who responded to initial TX chemotherapy. The current study indicated that single-agent capecitabine maintenance treatment was well tolerated and its ability to be orally administered avoids the need for a central venous device, thus, reducing discomfort and the risk of developing a central venous catheter infection. Furthermore, the use of oral capecitabine reduces the hospitalization and administration costs and appears to improve the patient quality of life.

In conclusion, the results of the present study indicate that single-agent capecitabine maintenance therapy may be an a potential therapeutic strategy for MBC patients who have responded to capecitabine-based chemotherapy prior to disease progression. In particular, capecitabine may offer a more effective maintenance treatment duration compared with HRT for patients who have previously undergone first-line palliative HRT for MBC.

\section{Acknowledgements}

The authors thank Dr Youyong Li of the Beijing Institute for Cancer Research, Professor Xing Zhou of Peking University and Professor Xingjie Liang of the National Center for Nanoscience and Technology for reviewing the manuscript. 


\section{References}

1. Siegel R, Naishadham D and Jemal A: Cancer statistics, 2012. CA Cancer J Clin 62: 10-29, 2012.

2. Thun MJ, Jemal A and Ward E: Global cancer incidence and mortality. In: Cancer: Principles and Practice of Oncology. DeVita VT Jr (ed). 9th edition. Lippincott Williams \& Wilkins, Philadelphia, PA, pp241-266, 2011.

3. Gennari A, Amadori D, De Lena M, et al: Lack of benefit of maintenance paclitaxel in first-line chemotherapy in metastatic breast cancer. J Clin Oncol 24: 3912-3918, 2006.

4. Alba E, Ruiz-Borrego M, Margelí M, et al: Maintenance treatment with pegylated liposomal doxorubicin versus observation following induction chemotherapy for metastatic breast cancer: GEICAM 2001-01 study. Breast Cancer Res Treat 122 169-176, 2010

5. Gennari A, Stockler M, Puntoni M, et al: Duration of chemotherapy for metastatic breast cancer: a systematic review and meta-analysis of randomized clinical trials. J Clin Oncol 29: 2144-2149, 2011

6. Dufresne A, Pivot X, Tournigand C, et al: Maintenance hormonal treatment improves progression free survival after a first line chemotherapy in patients with metastatic breast cancer. Int J Med Sci 5: 100-105, 2008

7. Bertelli G, Garrone O, Bertolotti L, et al: Maintenance hormone therapy with letrozole after first-line chemotherapy for advanced breast cancer. Oncology 68: 364-370, 2005.

8. Mayer IA: Treatment of HER2-positive metastatic breast cancer following initial progression. Clin Breast Cancer 9 (Suppl 2): S50-S57, 2009.

9. Leonard R, Hennessy BT, Blum JL and O'Shaughnessy J: Dose-adjusting capecitabine minimizes side effects while maintaining efficacy: a retrospective review of capecitabine for metastatic breast cancer. Clin Breast Cancer 11: 349-356, 2011.

10. Górnaś M and Szczylik C: Oral treatment of metastatic breast cancer with capecitabine: what influences the decision-making process? Eur J Cancer Care (Engl) 19: 131-136, 2010.

11. Reichardt P, Von Minckwitz G, Thuss-Patience PC, et al: Multicenter phase II study of oral capecitabine (Xeloda ${ }^{\circledR}$ ) in patients with metastatic breast cancer relapsing after treatment with a taxane-containing therapy. Ann Oncol 14: 1227-1233, 2003.

12. Kusama M, Nomizu T, Aogi K, et al: Phase II study of 4-weekly capecitabine monotherapy in advanced/metastatic breast cancer. Breast Cancer 17: 233-240, 2010.

13. Hortobagyi GN, Gomez HL, Li RK, et al: Analysis of overal survival from a phase III study of ixabepilone plus capecitabine versus capecitabine in patients with MBC resistant to anthracyclines and taxanes. Breast Cancer Res Treat 122: 409-418, 2010.

14. O'Shaughnessy J, Miles D, Vukelja S, et al: Superior survival with capecitabine plus docetaxel combination therapy in anthracycline-pretreated patients with advanced breast cancer: phase III trial results. J Clin Oncol 20: 2812-2823, 2002.

15. Blum JL, Dees EC, Vukelja SJ, et al: Phase II trial of capecitabine and weekly paclitaxel in patients with metastatic breast cancer previously treated with every-3-week taxane therapy. Clin Breast Cancer 7: 465-470, 2007.

16. Chan A and Verrill M: Capecitabine and vinorelbine in metastatic breast cancer. Eur J Cancer 45: 2253-2265, 2009.

17. Eisenhauer EA, Therasse P, Bogaerts J, et al: New response evaluation criteria in solid tumours: revised RECIST guideline (version 1.1). Eur J Cancer 45: 228-247, 2009.
18. Oken MM, Creech RH, Tormey DC, et al: Toxicity and response criteria of the Eastern Cooperative Oncology Group. Am J Clin Oncol 5: 649-655, 1982.

19. Trotti A, Colevas AD, Setser A, et al: CTCAE v3.0: development of a comprehensive grading system for the adverse effects of cancer treatment. Semin Radiat Oncol 13: 176-181, 2003.

20. Nicolini A, Giardino R, Carpi A, et al: Metastatic breast cancer: an updating. Biomed Pharmacother 60: 548-556, 2006

21. Cardoso F, Bedard PL, Winer EP, et al; ESO-MBC Task Force: International guidelines for management of metastatic breast cancer: combination vs. sequential single-agent chemotherapy. J Natl Cancer Inst 101: 1174-1181, 2009.

22. Park YH, Jung KH, Im SA, et al: Phase III, multicenter, randomized trial of maintenance chemotherapy versus observation in patients with metastatic breast cancer after achieving disease control with six cycles of gemcitabine plus paclitaxel as first-line chemotherapy: KCSG-BR07-02. J Clin Oncol 31: 1732-1739, 2013

23. Martín M and López-Tarruella S: Chemotherapy: Maintenance therapy in breast cancer - many questions remain. Nat Rev Clin Oncol 10: 370-372, 2013.

24. Sledge GW Jr, Hu P, Falkson G, Tormey D and Abeloff M: Comparison of chemotherapy with chemohormonal therapy as first-line therapy for metastatic, hormone-sensitive breast cancer: an Eastern Cooperative Oncology Group Study. J Clin Oncol 18: 262-266, 2000.

25. Beaver JA and Park BH: The BOLERO-2 trial: the addition of everolimus to exemestane in the treatment of postmenopausal hormone receptor-positive advanced breast cancer. Future Oncol 8: 651-657, 2012.

26. Ali S and Coombes RC: Endocrine-responsive breast cancer and strategies for combating resistance. Nat Rev Cancer 2: 101-112, 2002.

27. Burstein HJ: Novel agents and future directions for refractory breast cancer. Semin Oncol 38 (Suppl 2): S17-S24, 2011.

28. Mouridsen H, Sun Y, Gershanovich M, et al: Superiority of letrozole to tamoxifen in the first-line treatment of advanced breast cancer: evidence from metastatic subgroups and a test of functional ability. Oncologist 9: 489-496, 2004.

29. Nabholtz JM, Buzdar A, Pollak M, et al: Anastrozole is superior to tamoxifen as first-line therapy for advanced breast cancer in postmenopausal women: results of a north american multicenter randomized trial. Arimidex Study Group. J Clin Oncol 18: 3758-3767, 2000.

30. Mauri D, Pavlidis N, Polyzos NP and Ioannidis JP: Survival with aromatase inhibitors and inactivators versus standard hormonal therapy in advanced breast cancer: meta-analysis. J Natl Cancer Inst 98: 1285-1291, 2006.

31. Falkson G, Gelman RS, Pandya KJ, et al: Eastern Cooperative Oncology Group randomized trials of observation versus maintenance therapy for patients with metastatic breast cancer in complete remission following induction treatment. J Clin Oncol 16: 1669-1676, 1998.

32. Nooij MA, de Haes JC, Beex LV, et al; EORTC Breast Cancer Group: Continuing chemotherapy or not after the induction treatment in advanced breast cancer patients: Clinical outcomes and oncologists' preferences. Eur J Cancer 39: 614-621, 2003. 